МИНИСТЕРСТВО ОБРАЗОВАНИЯ И НАУКИ РОССИЙСКОЙ ФЕДЕРАЦИИ

ТОМСКИЙ ГОСУДАРСТВЕННЫЙ УНИВЕРСИТЕТ

\title{
МАТЕРИАЛЫ
}

VIII Международной молодежной научной конференции

«МАТЕМАТИЧЕСКОЕ

И ПРОГРАММНОЕ ОБЕСПЕЧЕНИЕ

ИНФОРМАЦИОННЫХ, ТЕХНИЧЕСКИХ

И ЭКОНОМИЧЕСКИХ СИСТЕМ»

Томск, 26-30 мая 2021 г.

Под общей редакиией И.С. Шмырина

Томск

Издательство Томского государственного университета 2021 


\title{
ЛИТЕРАТУРА
}

1. Koenigsegg C. Door hinge. [Электронный pecypc] // Google Patents. URL: https://patents.google.com/patent/US10662687B2/en (дата обращения: 11.06.2021).

2. The Making of Forza Motorsport 4: Car Sourcing [Электронный ресурс] // Официальный ҮоuТube канал Turn 10 Studios. URL: https://www.youtube.com/watch?v=CWb7cCS16TM (дата обращения: 11.06.2021).

3. Характеристики Koenigsegg One:1 [Электронный ресурс] // Официальный сайт Koenigsegg. URL: https://www.koenigsegg.com/car/one1/ (дата обращения: 11.06.2021).

4. Oliva M. Rear Cross Frame Re-Design and Optimization in Carbon Fiber. [Электронный ресурс] // База знаний Политехнического университета Каталонии. URL: https://upcommons.upc.edu/handle/2099.1/24276 (дата обращения: 11.06.2021).

5. Sköld O. The 3D-printed Koenigsegg Agera RS [Электронный pecypc] // The blog of Olle Sköld. URL: http://depronized.com/2019/04/29/the-3d-printed-koenigsegg-agera-rs/ (дата обращения: 11.06.2021).

6. Положение о проведении Фестиваля "Hello, Robot". [Электронный ресурс] // Официальный сайт центра "Планирование карьеры". URL: http://cpc.tomsk.ru/priglashaem-k-uchastiyu-v-festivale-po-obrazovatelnoyrobototehnike-hello-robot/ (дата обращения: 11.06.2021).

7. Дорожкин А.Е., Дорожкин Е.П., Изюмов А.А. Использование набора Lego Mindstorms 9797 для решения задачи "цветная сортировка" // Научная сессия ТУСУР-2017 : материалы Международной научно-технической конференции студентов, аспирантов и молодых ученых, посвященной 55-летию ТУСУРа. Томск, 10-12 мая 2017 г. - В-Спектр, 2017. - Ч. 1. - С. 263-266.

DOI: $10.17223 / 978-5-907442-42-9-2021-2$

\section{ДЕТЕКТИРОВАНИЕ СТАНДАРТНЫХ ДОРОЖНЫХ ЗНАКОВ НА ИЗОБРАЖЕНИЯХ}

\author{
Рудов В.А., Приступа А.В., Скворцов А.В. \\ Томский государственный университет \\ rudov.vlad2013@gmail.com, andreypr@mail.ru
}

\section{Введение}

Задача распознавания дорожных знаков на фото- и видеоизображениях достаточно часто возникает в самых разных отраслях: в дорожном и железнодорожном хозяйстве при паспортизации и оценке уровня содержания дорог, в беспилотных автомобилях для коррекции цифровой модели дороги при движении по заданному навигационному маршруту [1].

После принятия в 1968 г. Венской конвенции о дорожном движении основная масса знаков в мире была стандартизирована [1], и только небольшое число знаков допускалось оформлять нестандартно текстовыми надписями и стрелками. Такие знаки в России называются знаками индивидуального проектирования (ЗИП) [2]. И если распознавание таких знаков ещё алгоритмически возможно, то их автоматизированная интерпретация весьма сомнительна. Именно поэтому в основном решается задача распознавания стандартных знаков.

Существующие методы решения задачи детектирования объектов на изображении можно разделить на 3 категории [3]:

- опирающиеся на цветовые признаки;

- опирающиеся на форму и контур;

- на основе нейронных сетей.

За последние годы методы, опирающиеся на цветовые признаки, форму и контур объектов, в реальных условиях показали свою недостаточно высокую эффективность, Т.К.:

- объект на изображении может находиться не под прямым углом или может оказаться повернут вокруг своей оси;

- цвета на изображении могут быть искажены ввиду различных погодных условий или освещения;

- знак может частично перекрываться другим объектом. 
Именно поэтому основные исследования последнего десятилетия нацелены на применение нейронных сетей: как правило, исследователи предлагают различные варианты свёрточных нейронных сетей.

Задача распознавания знаков обычно решается в два этапа: поиск областей на изображении с потенциальными знаками (задача сегментации) и распознавание знака в указанной области (задача идентификации).

В свою очередь, т.к. знаки имеют разную форму (круг, треугольник, квадрат, ромб) и основной цвет (белый, синий, жёлтый), то уже на этапе сегментации можно выделять регионы соответствующей формы (например, региональными свёрточными нейронными сетями серии R-CNN [4]) и существенно сужать последующий поиск в задаче идентификации.

В настоящей работе предлагается единый алгоритм, последовательно решающий всю задачу распознавания стандартных дорожных знаков: от предварительной подготовки изображения с помощью OpenCV [5], предварительного поиска областей методом максимально стабильных экстремальных областей (MSER) [6] и их фильтрации до последующей идентификации знаков с помощью сети ResNet [7]. Для сравнения полученного решения с аналогами использовался набор реальных изображений, а также автоматически подготовленные синтетические изображения знаков.

\section{1. Предлагаемый алгоритм}

Общий алгоритм решения поставленной задачи в общем виде представлен на рис. 1. На вход подаётся изображение дороги, которое после предварительной обработки поступает на вход детектора MSER для сегментации изображения. Полученные на выходе детектора регионы проходят фильтрацию и поступают на вход свёрточной нейронной сети, которая выделяет лишь те регионы, которые содержат дорожный знак.

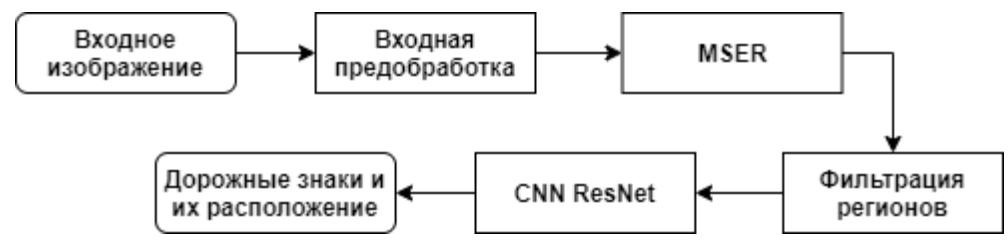

Рис. 1. Схема работы алгоритма детектирования

Для увеличения контрастности входного изображения используется алгоритм улучшения контрастности CLAHE (Contrast Limited Adaptive Histogram Equalization) [8]. В отличие от других алгоритмов он более устойчив к порождению излишнего шума, при этом его реализация доступна в библиотеке OpenCV.

Детектор MSER выделяет множество различных регионов с экстремальными свойствами функции интенсивности внутри региона и на его внешней границе.

В статье Кристиана Миколайджека [9] приведён сравнительный анализ шести детекторов областей, в том числе и детектора MSER, показавший, что:

- MSER обнаруживает наибольшее множество небольших областей при изменении точки обзора;

- MSER занял второе место по обнаружению при изменении масштаба и вращению в плоскости;

- MSER продемонстрировал наибольшую повторяемость результатов при изменении освещения сцены.

Таким образом, для предварительного поиска областей, потенциально содержащих дорожных знак, в данной работе принято решение использовать OpenCV реализацию MSER-детектора.

Одним из недостатков детектора MSER является обнаружение достаточно большого количества регионов. Чтобы отсеять области, не содержащие дорожные знаки, был 
разработан фильтр выходных регионов. Из выделенных MSER областей отбирались лишь те, соотношение сторон которых не превышает 1.5 (т.е. достаточно близкие по форме к квадрату; в действительности же есть знаки с другими пропорциями, но к ним требуется иной подход), а их длина и ширина лежит в пределах от 35 до 200 пикселей. Так отсеиваются области некорректной формы (слишком малые или слишком крупные для дорожного знака.

Пример работы этапа фильтрации представлен на рис. 2. В приведённом примере алгоритмом MSER было выделено 588 областей, а после применения фильтрации осталось 159 областей. Таким образом, применение выходной фильтрации может уменьшить количество выходных регионов в разы.
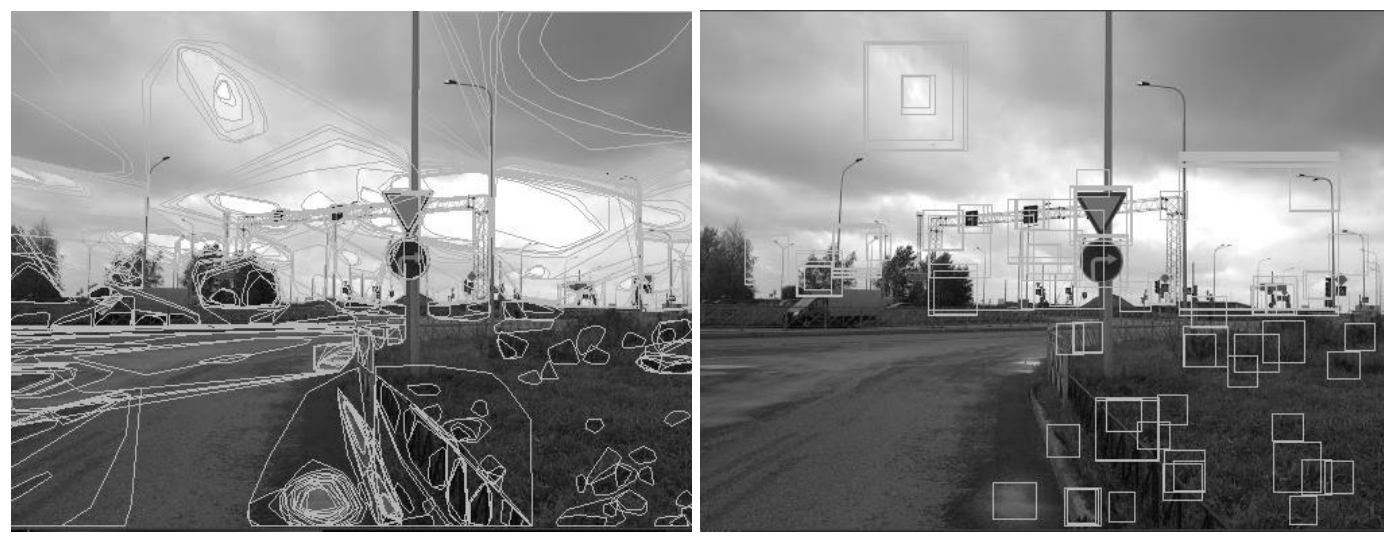

Рис. 2. Результат сегментации изображения на регионы алгоритмом MSER

После работы алгоритма MSER сегментированные регионы необходимо классифицировать. В данной работе предлагается использовать ResNet (Residual Network «остаточная сеть») - уже классическая свёрточная нейронная сеть, используемая в качестве основы для многих задач компьютерного зрения.

Основная идея нейронной сети ResNet заключается в добавлении так называемых «соединений быстрого доступа» - связей между несмежными слоями (рис. 3). При этом обучение такой сети занимает большее количество времени, но и растёт точность классификации при существенном увеличении глубины.

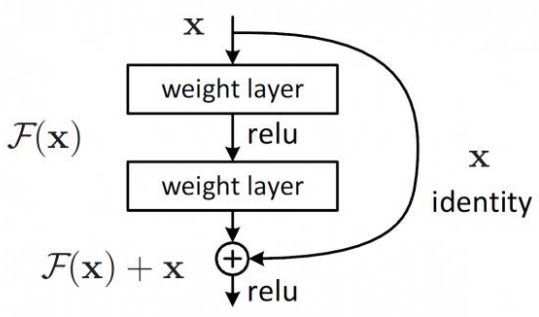

Рис. 3. Соединения быстрого доступа

Существует несколько классических разновидностей сетей ResNet, которые отличаются числом слоёв. Для исследовательских целей (для распознавания небольшого подмножества типов знаков) нами была выбрана сеть ResNet34: она сходится быстрее, чем более глубокая ResNet50, и при этом обеспечивает лучший результат обучения по сравнению с более мелкой сетью ResNet18. При реализации же коммерческого решения (для распознавания сотен видов и подвидов знаков) более предпочтительным является использование ResNet101 или ResNet152. 


\section{2. Тестовые наборы данных}

Для проверки работоспособности предлагаемого подхода было выбрано подмножество знаков (табл. 1), которые будут распознаваться. Среди них можно выделить как группы знаков, имеющие схожие признаки (круглые, квадратные, треугольные), так и уникальные знаки (ромб, перевёрнутый треугольник).

Таблица 1

Типы детектируемых дорожных знаков

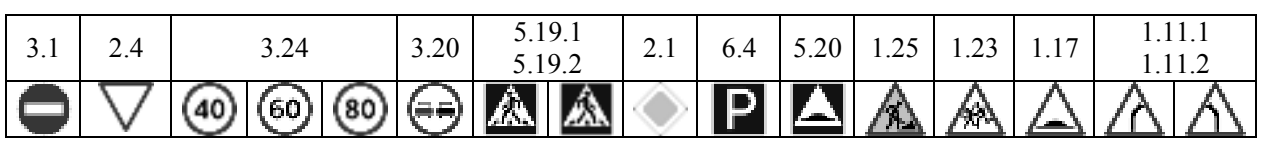

Для обучения нейронной сети использовались реальные (фотографии дорожных знаков) и синтетические данные (программно сгенерированные изображения).

В качестве реальной основы для обучения и тестирования сверточной нейронной сети взят набор российских дорожных знаков (Russian Traffic Sign Dataset, RTSD). Эта выборка содержит кадры, снятые в различных условиях освещения, погодных условиях и временах года, и содержит 156 классов знаков [10]. На рис. 4 представлены примеры данных из RTSD.

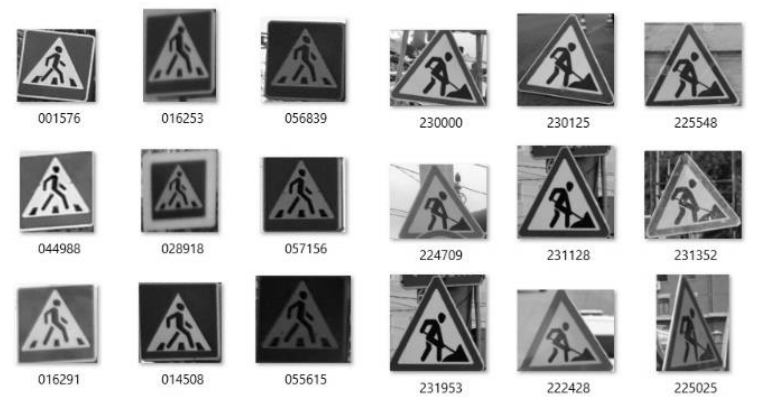

Рис. 4. Примеры знаков 5.19.2 и 1.25 из RTSD

Из всего набора данных RTSD были выделены только требуемые для реализации системы классы дорожных знаков. С целью оценки качества используемого набора данных была построена гистограмма распределения его тренировочных и тестовых данных (рис. 5).

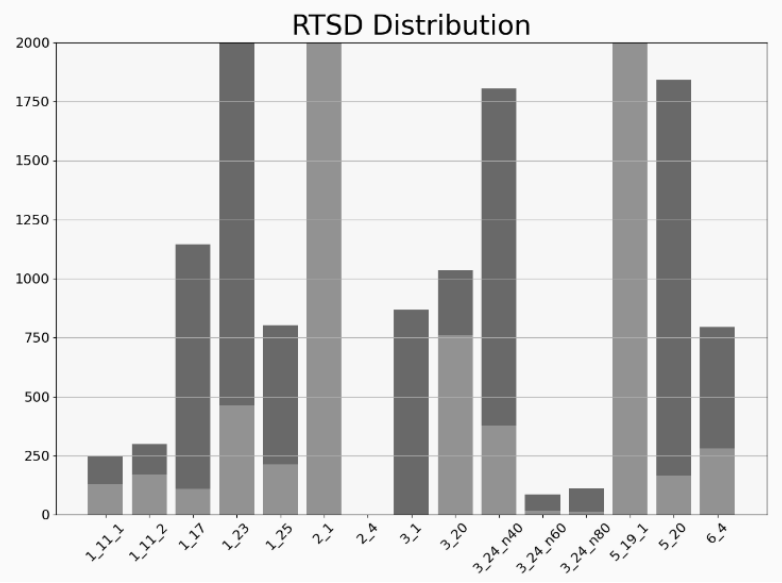

Рис. 5. Диаграмма распределения данных по классам в исходном наборе RTSD, темным цветом обозначены тренировочные данные, светлым - тестовые 
Полученное распределение образцов оказалось неравномерным: некоторые данные отсутствуют, а количество изображений знаков 3.24 (60 шт.) и 3.24 (80 шт.) критически мало. Для решения этой проблемы было решено дополнить группы с наименьшим содержанием дорожных знаков (менее 600 шт.) реальными данными из сторонних источников и синтетическими данными, а также удалить образцы размером менее $35 \times 35$ пикселей. После проделанных манипуляций распределение данных приняло следующий вид (рис. 6).

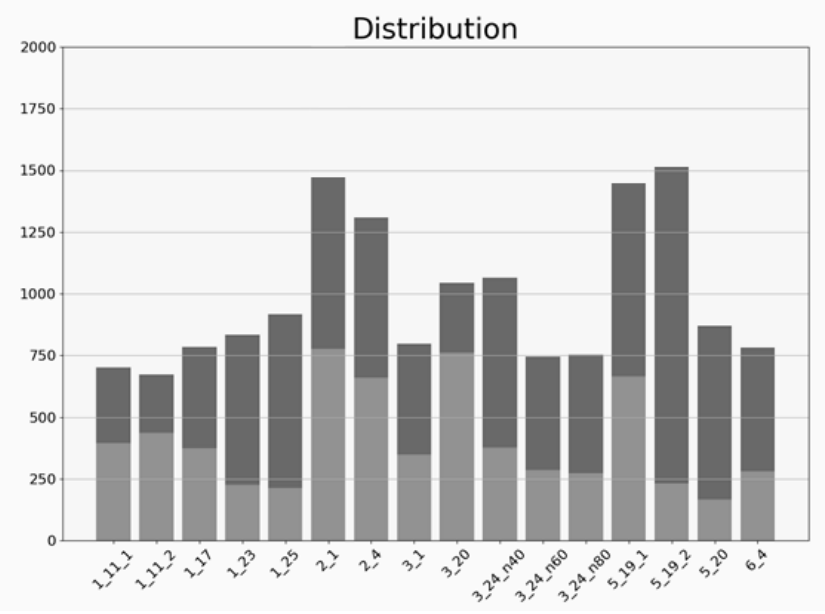

Рис. 6. Конечное распределение используемого набора данных

Полученное распределение является относительно равномерным: в каждом классе присутствует минимум 600 образцов.

Дополнительно в датасет были добавлены изображения, не содержащие дорожных знаков (отрицательные прецеденты). Для генерации таких областей использовалась следующая процедура:

1) к набору изображений из тренировочных данных применялся детектор MSER c последующей фильтраций регионов;

2) среди полученных регионов выбирались области, не содержащие дорожный знак, но при этом находящиеся относительно недалеко от него.

Количество таких изображений составило около $20 \%$ от общего объёма выборки.

Для дополнения существующей выборки была сгенерирована вспомогательная синтетическая выборка дорожных знаков. В качестве основы для генерации был использован набор векторных изображений дорожных знаков размером $512 \times 512$ пикселей из специализированной системы проектирования знаков дорожного движения IndorRoadSigns [11]. К этим векторным изображениям применялись следующие преобразования:

1) аффинные преобразования и изменение перспективы (рис. 7);

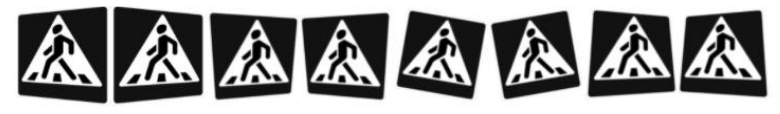

Рис. 7. Применение аффинных и проективных преобразований

2) добавление случайного фона, вырезанного из реальных изображений (рис. 8);

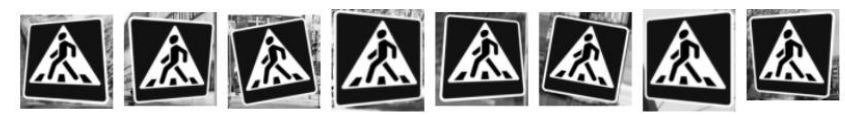

Рис. 8. Примеры изображений с добавленным фоном 
3) различного рода искажения, такие, как размытие по Гауссу, размытие в движении; гауссовский шум, эффект дождя, эффект тумана, изменение яркости компонент изображения в цветовом пространстве HSV (рис. 9).

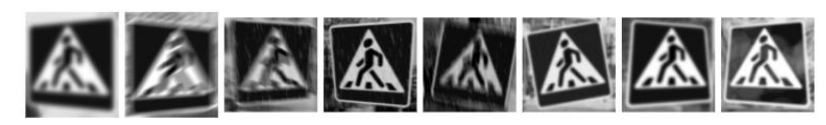

Рис. 9. Примеры изображений с искажениями

Идея применения именно этих способов искажения обусловлена исследованием Шахуро В.И. [12], где полученная на их основе синтетическая выборка показала достойные результаты. Сгенерированная нами синтетическая выборка состоит из 16 классов знаков и включает в себя $350 \times 16=5600$ изображений.

\section{3. Реализация свёрточной нейронной сети ResNet34 на Keras}

C помощью библиотеки Keras на языке Рython нами была реализована архитектура сети ResNet34 со следующими изменениями:

1) выходной слой 1000-d fc SoftMax был заменён на полносвязный выходной слой 17-d fc SoftMax. Данная размерность соответствует размерности заданного множества для классификации (16 классов дорожных знаков и 1 класс отрицательных прецедентов);

2) в модуль обучения нейронной сети были добавлены планировщик скорости обучения, который помогает модели лучше учиться, и проверка контрольной точки (сохранение модели с наилучшей точностью).

После задания архитектуры полученная модель была скомпилирована со следующими параметрами:

1) метод оптимизации: Adam;

2) функция потерь: categorical_crossentropy.

Перед использованием изображений в качестве входных данных необходимо произвести их предварительную обработку. Для этого использовался метод библиотеки Keras: tf.keras.preprocessing.image_dataset_from_directory [13]. Данный метод позволяет перейти от необработанных данных на диске к объекту tf.data.Dataset, который можно использовать для обучения модели. В результате изображения из выборок были приведены к размеру 96×96 пикселей, перемешаны и разделены на партии по 16 экземпляров. Кроме того, из обучающей выборки было выделено 20\% данных для выборки валидации, чтобы оценить ожидаемый уровень соответствия модели.

Затем была произведена нормализация значений тензора из интервала от 0 до 255 к интервалу от 0 до 1, т.к. нейронные сети лучше работают с маленькими входными значениями. Обучение производилось в течение 35 эпох и заняло около 1 часа. График процесса обучения представлен на рис. 10.

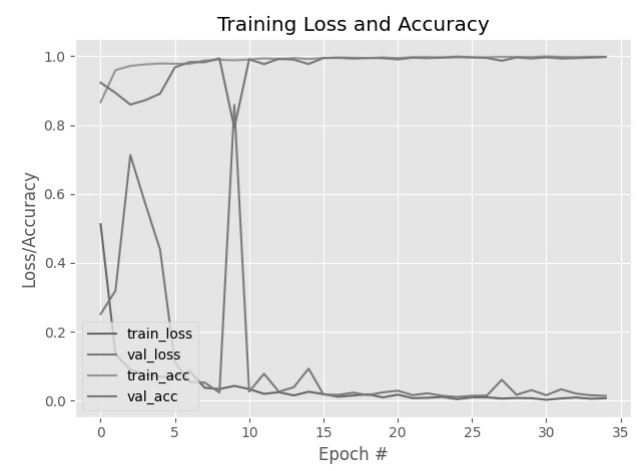

Рис. 10. График обучения нейронной сети 
Достигнутая точность на тестовой выборке составила 0,96, что является достаточно хорошим результатом. После обучения модель была сохранена в формате двоичных данных HDF5 и в дальнейшем может использоваться без необходимости обучать её снова.

\section{4. Тестирование и оценка точности}

Для того чтобы оценить результат работы системы, было произведено её тестирование на изображениях реальных дорожных сцен. При этом производился подсчёт характеристик False negative (число случаев, когда алгоритм не выделил дорожные знаки, а они были) и False positive (число случаев, когда алгоритм выделил дорожные знаки там, где их не было).

Количество тестовых изображений составило 100 шт., на которых изображено 187 знаков дорожного движения. Результаты тестирования представлены в табл. 2.

Результаты тестирования

Таблица 2

\begin{tabular}{|c|c|c|c|}
\hline Общее количество & Детектировано корректно & False negative & False positive \\
\hline 187 & 173 & 14 & 16 \\
\hline
\end{tabular}

Полученные результаты свидетельствуют о том, что предлагаемый алгоритм вполне пригоден для практического применения. Ниже представлено несколько характерных примеров работы алгоритма (рис. 11.1 - 11.6).

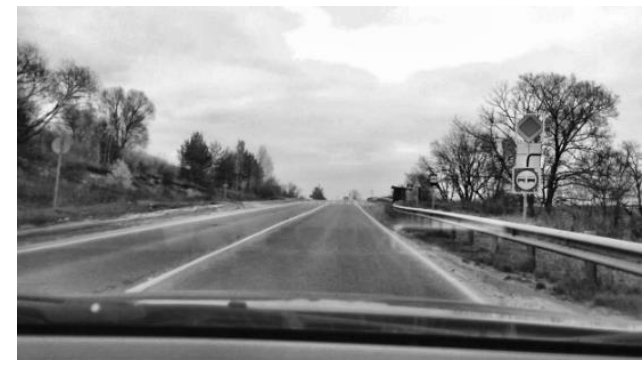

Рис. 11.1. Все знаки локализованы корректно

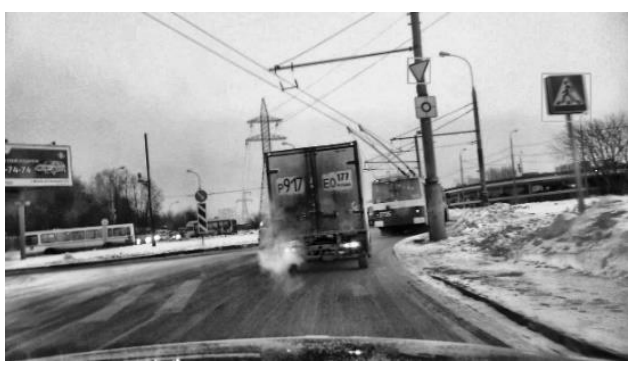

Рис. 11.3. Знаки, расположенные под углом, локализованы корректно

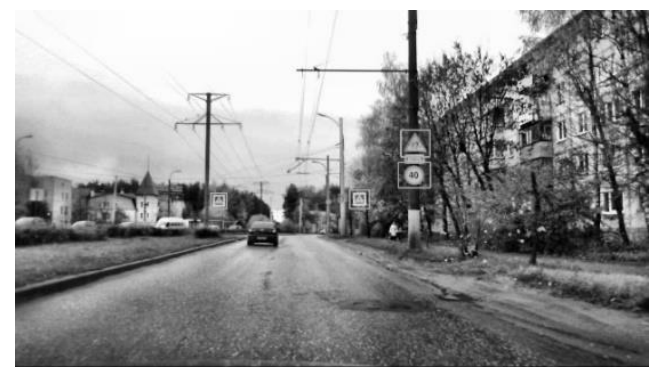

Рис. 11.2. Большое количество знаков локализовано корректно

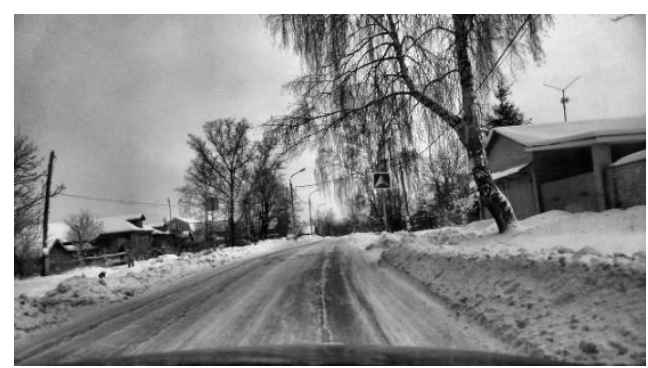

Рис. 11.4. Знак, заслонённый препятствием, локализован корректно 


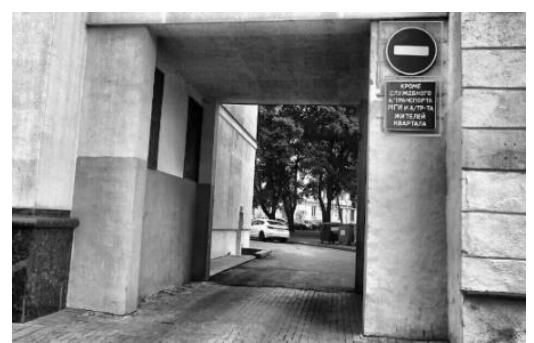

Рис. 11.5. Вместе со знаком 3.1 локализован объект, схожий с дорожным знаком

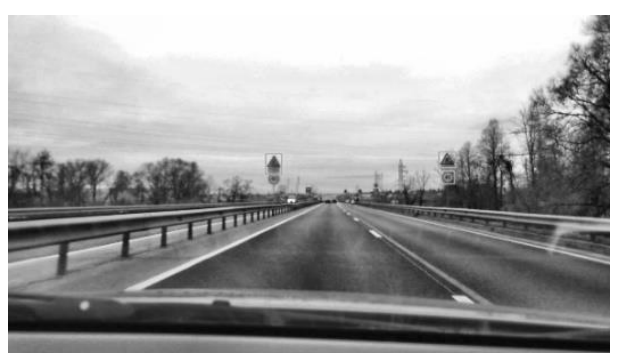

Рис. 11.6 - Алгоритм локализовал не все изображенные знаки

\section{Заключение}

В работе предложен способ автоматического распознавания стандартных дорожных знаков, основанный на совместном применении алгоритма сегментации областей MSER и детектирования знаков свёрточной нейронной сетью ResNet. Для обучения нейронной сети использован открытый набор данных RTSD и собственный синтезированный набор изображений дорожных знаков с различными искажениями. Для этого в специализированном редакторе дорожных знаков IndorRoadSigns создавались эталонные изображения, а на их основе генерировались различные синтетические изображения с помощью аффинных и проективных преобразований, различных растровых эффектов, а также добавления случайного заднего фона, вырезанного из реальных изображений.

Полученный программный модуль способен определять на изображениях некоторые стандартные знаки дорожного движения из указанного в работе списка в различных условиях. Для детектирования большего количества знаков обучение нейросети необходимо проводить на большем наборе данных, включающем в себя недостающие знаки. Для распознавания нестандартных знаков индивидуального проектирования требуются иные подходы, выходящие за рамки данной статьи.

\section{ЛИТЕРАТУРА}

1. Венская конвенция о дорожном движении. - 1968. - 19 с.

2. ГОСТ 52290-2004. Технические средства организации дорожного движения. Знаки дорожные. Общие технические требования. - $194 \mathrm{c}$.

3. Saadna Y., Behloul A. An overview of traffic sign detection and classification methods. // Int. J. Multimed Info Retr. - 2017. - V. 6. - P. 193-210.

4. Gandhi R. R-CNN, Fast R-CNN, Faster R-CNN, YOLO - Object Detection Algorithms // Towards Data Science. -2018 . - July 9.

5. OpenCV [Электронный ресурс] // URL: http://www.opencv.org

6. Matas J., Chum O., Urban M., Pajdla T. Robust wide-baseline stereo from maximally stable extremal regions // Image and Vision Computing. - 2004. - V. 22(10). - P. 761-767.

7. He K., Zhang X.; Ren Sh., Sun J. Deep Residual Learning for Image Recognition". arXiv: 1512.03385. 2015-1210.

8. Pizer S.M., Amburn E.P., Austin J.D. et al. Adaptive Histogram Equalization and Its Variations // Computer Vision, Graphics and Image Processing. - 1987. - V. 39. - P. 355-368.

9. Mikolajczyk K., Tuytelaars T., Schmid C., Zisserman A., Matas J., Schaffalitzky F., Kadir T., Gool L.V. A comparison of affine region detectors // International Journal of Computer Vision. - 2005. - V. 65. - P. 43-72. P. 294-300

10. Shakhuro V.I., Konushin A.S. Russian traffic sign images dataset // Computer Optics. - 2016. - V. 40. - № 2. -

11. Система проектирования дорожных знаков IndorRoadSigns [Электронный pecypc] // URL: https://indorsoft.ru/products/roadsigns

12. Шахуро В.И. Распознавание редких дорожных знаков с использованием синтетических обучающих выборок: диссертация на соискание учёной степени кандидата компьютерных наук. Высшая школа экономики, Москва, 2020. URL: https://www.hse.ru/sci/diss/426009543

13. TensorFlow image_dataset documentation [Электронный pecypc] URL:https:/www.tensorflow.org/api_docs/python/tf/keras/preprocessing/image_dataset_from_directory 